\title{
Growth mechanism and joint structure of ZnO tetrapods
}

\author{
Bin-Bin Wang, Ji-Jia Xie, Quanzi Yuan and Ya-Pu Zhao ${ }^{1}$ \\ State Key Laboratory of Nonlinear Mechanics, Institute of Mechanics, Chinese Academy of Sciences, \\ Beijing 100190, People's Republic of China \\ E-mail: yzhao@imech.ac.cn
}

Received 16 March 2008

Published 22 April 2008

Online at stacks.iop.org/JPhysD/41/102005

\begin{abstract}
Regular zinc oxide $(\mathrm{ZnO})$ tetrapods with a flat plane have been obtained on $\mathrm{Si}(100)$ substrate via the chemical vapour deposition approach. The $\mathrm{x}$-ray diffraction result suggests that these tetrapods are all single crystals with a wurtzite structure that grow along the $\left(\begin{array}{lll}0 & 0 & 0\end{array}\right)$ direction and corresponding electron backscatter diffraction analysis reveals the crystal orientation of growth and exposed surface. Furthermore, we find some $\mathrm{ZnO}$ tetrapods with some legs off and the angles between every two legs are measured with the aid of scanning electron microscopy and image analysis, which benefit to reveal the structure of $\mathrm{ZnO}$ tetrapods joint. The structure model and growth mechanism of $\mathrm{ZnO}$ tetrapods are proposed. Besides, the stable model of the interface was obtained through the density-functional theory calculation and the energy needed to break the twin plane junction was calculated as $5.651 \mathrm{~J} \mathrm{~m}^{-2}$.
\end{abstract}

\section{Introduction}

Zinc oxide $(\mathrm{ZnO})$ is a high-energy gap semiconductor of the II-IV group that has drawn considerable interest. ZnO nanomaterials possess remarkable optical, electrical magnetic and mechanical properties; thus they have prospective applications in transistors, sensors, etc. Such a popularity of this material explains the large number of studies dedicated to synthesis of one-dimensional (1D) or quasi-1D $\mathrm{ZnO}$ nanostructures. Naturally, shape and dimensionality play important roles in the properties and applications of nanomaterials. So far, various $\mathrm{ZnO}$ nanostructures with exciting morphologies, such as nanobelts [1], nanowires [2], nanosprings [3], nanorods [4], nanoflowers [5] and nanohelices [6] have been reported. Among these growth methods, chemical vapour deposition (CVD) technology is particularly interesting not only because it gives rise to high-quality films but also because it is applicable to large-scale production. This technique has been widely used in the fabrication of epitaxial films toward various GaN-based optoelectronic devices, and a similar trend might be expected for future applications of $\mathrm{ZnO}$.

\footnotetext{
${ }^{1}$ Author to whom any correspondence should be addressed.
}

The structure of $\mathrm{ZnO}$ tetrapods [6-8] also attracts much attention, which is important for the growth model of tetrapodlike $\mathrm{ZnO}$. Shiojiri and Kaito suggested a growth model in which the seed nucleus is a zinc-blende and a single-crystalline $\mathrm{ZnO}$ nanocrystal of octahedral shape with eight (1 111 ) surfaces [9]. Four of the surfaces are zinc terminated and act as nucleation sides for hexagonal $\mathrm{ZnO}$ crystals, which overgrow the nucleus resulting in $\mathrm{ZnO}$ tetrapods. Iwanaga et al introduced the common 'octa-twin model'. The main idea of this model is that the centre nucleus is an octahedral multiple inversion twin made up of eight trigonal pyramidal crystals with a $\left(\begin{array}{llll}0 & 0 & 0 & 1\end{array}\right)$ basal plane and three twin planes [10]. Zhang et al [11] proposed a twin plane structure of the tetrapods with the observation of scanning electron microscopy (SEM), and the SEM observation indicated that there were twin planes between every two legs and the tetrapods were of perfect tetrahedral symmetry [11].

In this communication, we employed the CVD method to synthesize the $\mathrm{ZnO}$ nanomaterials. The as-synthesized products are tetrapod-like and have large surface area. With the help of SEM and electron backscatter diffraction (EBSD) analysis, the structure of the junction of tetrapods is proposed. We find that not all $\mathrm{ZnO}$ tetrapods are of perfect symmetry, 
which could be attributed to the strain caused by lattice mismatch. Furthermore, based on the density-functional theory (DFT) calculation of the twin plane, we obtain the stable model of the interface, the surface energy of ( $\left.\begin{array}{lll}1 & 0 & 1\end{array}\right)$ and the interfacial energy. The results are helpful to the investigation of the interface formation and the mechanical strength of the joint.

\section{Experimental}

These regular $\mathrm{ZnO}$ tetrapods were synthesized by a simple catalyst-free CVD method. About $2.0 \mathrm{~g}$ of $\mathrm{Zn}$ powder $(99.999 \%)$ was evenly placed on the bottom of an alumina boat which was inserted into the centre of the quartz tube placed horizontally in a tubular furnace. A silicon substrate was laid face-down on the boat. When the desired temperature $\left(630^{\circ} \mathrm{C}\right)$ was reached, a flow of Ar at a rate of about 300 SCCM (SCCM denotes cubic centimetre per minute at standard temperature and pressure) and $\mathrm{O}_{2}$ at about $25 \mathrm{SCCM}$ was introduced into the system. After $30 \mathrm{~min}$, the flows of $\mathrm{Ar}$ and $\mathrm{O}_{2}$ were shut down, and the alumina boat was cooled down to room temperature in the protection of Ar ambience. Products were collected on the surface of the substrates. The as-prepared $\mathrm{ZnO}$ tetrapods were characterized using x-ray diffraction (XRD, D/MAX-RB), field emission scanning electron microscopy (FESEM FEI SIRION400 NC) equipped with energydispersive $x$-ray spectroscopy (EDS) and EBSD, highresolution field emission transmission electron microscopy (HR-FETEM, JEOL JEM-3010) equipped with selected area electron diffraction (SAED).

\section{Experimental results and discussion}

The EDS and XRD measurements were employed to investigate the composition and structure of the obtained samples. The EDS analysis shows that the synthesized sample is composed of $\mathrm{Zn}$ and $\mathrm{O}$ with no other elements detected.

The crystal structure of the $\mathrm{ZnO}$ tetrapods was examined with XRD as shown in figure 1 $(a)$. All peaks are sharp and strong, suggesting that the products are highly crystallized, and all the visible diffraction intensities agree well with the peaks of $\mathrm{ZnO}$ wurtzite structure with the lattice constants of $a=3.253 \AA$ and $c=5.209 \AA$. No intensities of zinc or other impurities are found in the spectrum, which also shows that the tetrapods are rather pure $\mathrm{ZnO}$ wurtzite.

Figure 1(b) shows the SAED pattern of the sample. The SAED pattern was taken from one of the legs which confirm that the product is a perfect single crystal with wurtzite structure and each leg grows along the $\left(\begin{array}{llll}0 & 0 & 0 & 1\end{array}\right)$ direction.

Although transmission electron microscopy (TEM) is the most powerful and widely used method for the crystallographic characterization of nanostructures, it is not ideal for a large sample because of the weak penetrability of large structures. For the sample in this work, EBSD is a valuable tool giving fast and accurate crystallographic information. Figure 2 shows that EBSD result. The growth orientation is confirmed to be

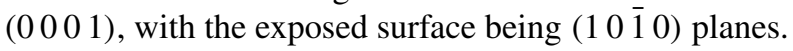

Figure 3(a) shows a typical SEM image of the synthesized $\mathrm{ZnO}$ tetrapods with the sample. The as-grown nanostructures
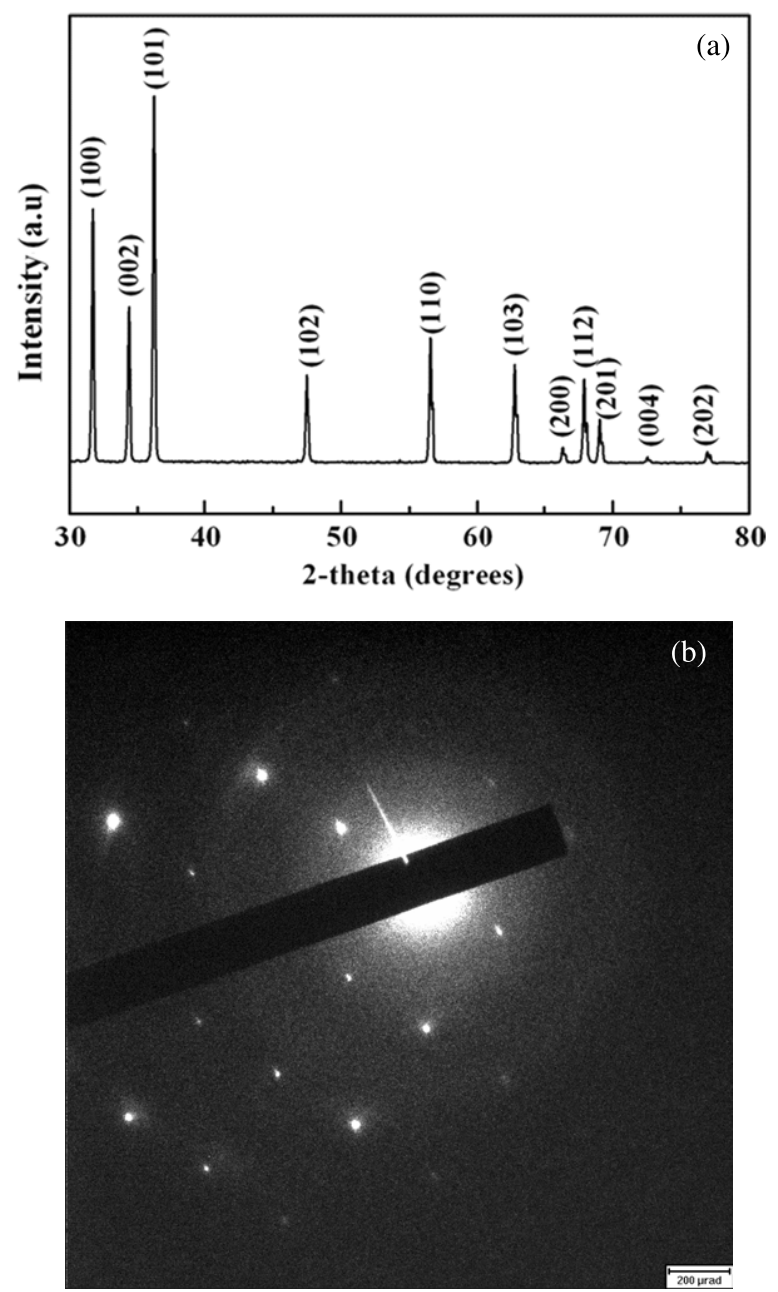

Figure 1. (a) XRD pattern of as-prepared $\mathrm{ZnO}$ tetrapods grown on Si substrate showing wurtzite structure and high crystallinity and (b) SAED pattern indicating the single-crystalline nature of $\mathrm{ZnO}$ tetrapods with the $(00001)$ orientation.

are uniform tetrapod-like. The leg of each structure is $1.5-3 \mu \mathrm{m}$ in length, with the diameter of about $300-700 \mathrm{~nm}$. Regular $\mathrm{ZnO}$ tetrapods are of tetrahedral symmetry and (0 0001 ) hexagonal planes are present at the end of the legs. Furthermore, there is a twin plane between every two legs, which means each arm has three twin planes. As the legs could easily break off from tetrapods junction along the twin planes, we could get many clear SEM images of the tetrapods with some legs off. Figure 3(b) shows the typical junction of the tetrapods. Figures $3(c)$ and $(d)$ directly show the existence of twin planes between every two legs, and the nucleus is not seen in the pictures. Figure 3(c) shows a typical single leg, exhibiting the broken part including the three planes. These planes are all of lozenge shape. Figure $3(d)$ shows that two of the arms are broken off from the junction and four lozenge planes are present clearly. The inhomogeneous surfaces in figure $3(d)$ possibly attribute to the influence of the growth condition fluctuation on the formation of the crystal surface. Figure 3(e) shows that one of the arms is broken off from the junction and three lozenge planes are present at the junction.

The regular $\mathrm{ZnO}$ tetrapods in this work were fabricated without the presence of any catalysts or other additives. It 

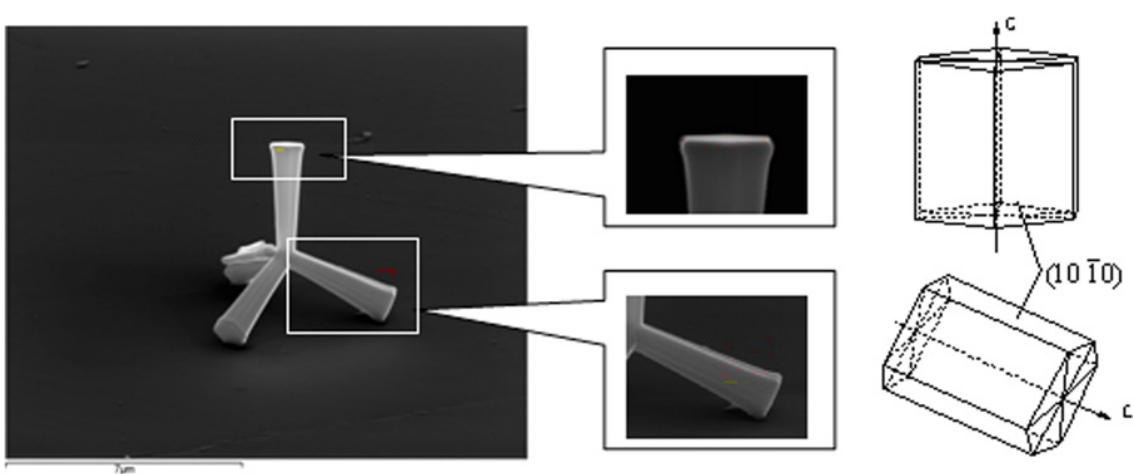

Figure 2. The EBSD investigation of a typical $\mathrm{ZnO}$ tetrapod is shown with the analysed area, showing that the growth direction of the nanowires is parallel to the $c$ axis. The schematic crystal indicates the directions of the crystal axes and the crystal orientation of exposed surface.

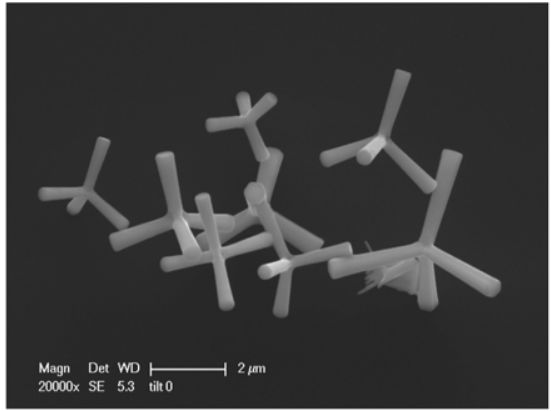

(a)

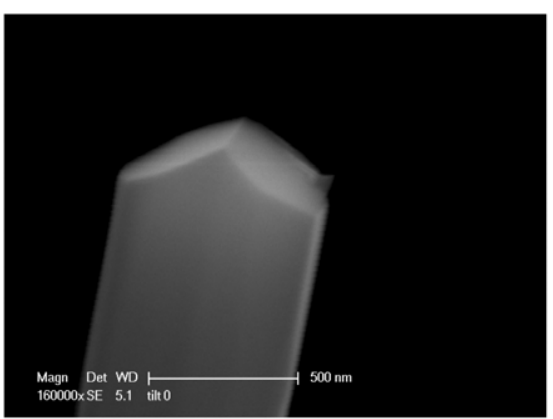

(c)

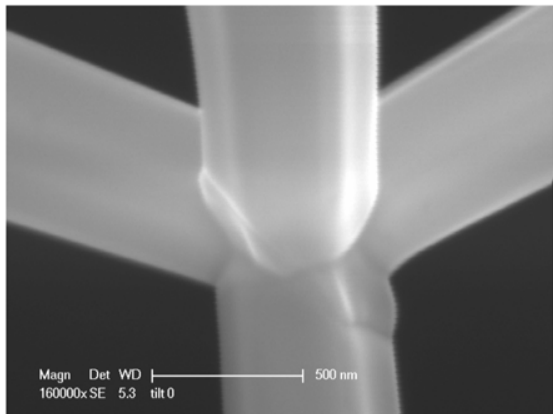

(b)

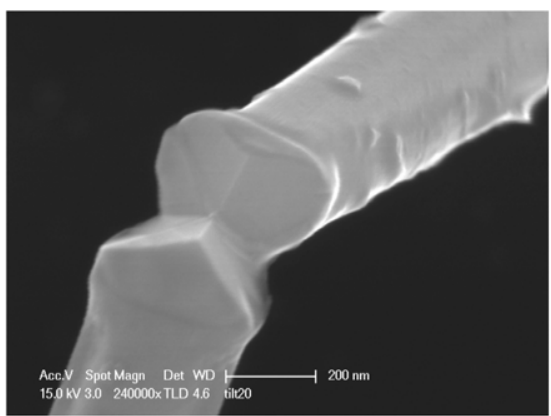

(d)

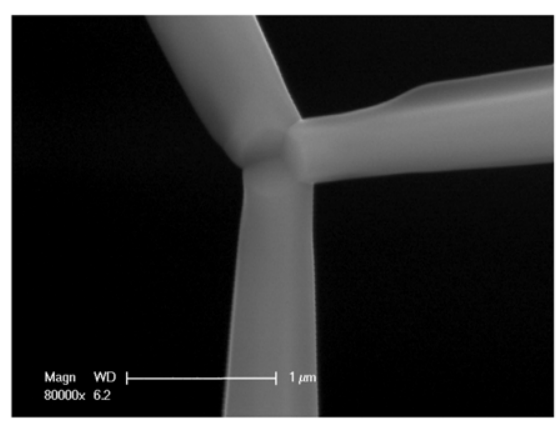

(e)

Figure 3. (a) SEM of ZnO tetrapods with flat plane on the top. (b)-(d) SEM of tetrapods with different arms: (b) The integral junction of a $\mathrm{ZnO}$ tetrapod, $(c)$ one single leg of a $\mathrm{ZnO}$ tetrapod, $(d)$ SEM of tetrapods with two legs broken off, $(e)$ SEM of tetrapods with one leg broken off.

is generally believed that $\mathrm{ZnO}$ tetrapods are governed by the vapour-solid (VS) process rather than by the vapourliquid-solid (VLS) model. In the VS mechanism, the $\mathrm{Zn}$ is self-catalyzed, while the typical characteristic of the VLS mechanism is that the synthesized materials have metal particles capped at the end of them [12]. Ronning and Shang proposed a nucleus model with the centre core of $\mathrm{ZnO}$ tetrapods consists of four hexagonal grains with a twin like relation as shown in figure 4(a) [13]. According to this model, the four hexagonal grains are nucleated as embryos during the 


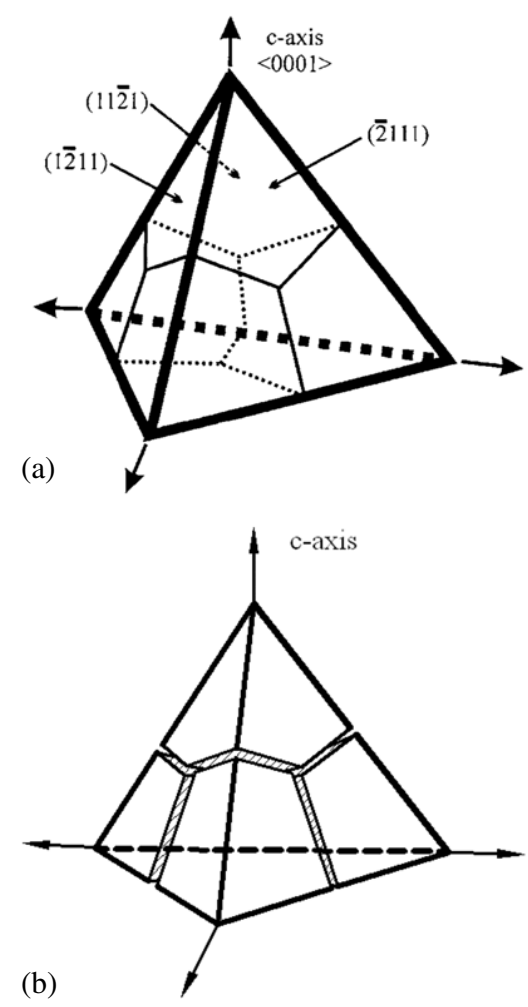

Figure 4. (a) Schematic model of nucleus structure [13] and (b) the decohesion of twin boundaries leads to strain relaxation.

initial growth stage. Each hexahedral crystal consists of lowindex, high-atomic-density planes, which are supposed to be

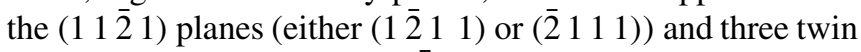
planes (for example, the (1 $\left.0 \begin{array}{lll}1 & \overline{1} & 3\end{array}\right)$ plane), and the crystals are connected to each other through these twin planes. The growth along the different directions of a $\mathrm{ZnO}$ crystal is quite different and preferentially grows along the $\left(\begin{array}{llll}0 & 0 & 0 & 1\end{array}\right)$ direction. Thus the $\mathrm{ZnO}$ crystals adopt a tetrapod shape. It is assumed that when the grains join together, a large lattice mismatch strain occurs. Owing to the large strain, the nucleus is stable only for finite small sizes, up to a point where the surface energy contribution to the total energy is meaningful [14]. When the nucleus grows to a size larger than a critical limit, they are no longer stable and could disjoint easily along the twin boundaries in order to relieve the strain energy accumulated at the interface (figure $4(b)$ ).

Furthermore, we have measured the angles between every two legs for the as-grown sample. We find that not all the $\mathrm{ZnO}$ tetrapods are of perfect symmetry. Figure 5(a) shows the image of one typical $\mathrm{ZnO}$ tetrapod under SEM, and figure $5(b)$ is the same structure with the sample stage tilted by $30^{\circ}$. To get the angles between every two legs of $\mathrm{ZnO}$ tetrapod, the projected angles of each branch of $\mathrm{ZnO}$ particles were firstly measured at two different stage tilt angles. Then, according to the geometrical relationship of these two teams of projected angles, angles between every two legs of $\mathrm{ZnO}$ particles can be acquired with the help of image analysis. The result is shown in table 1.

One possible reason for this asymmetry lies in the difference in the growth speed of each leg. In the early growth stage, the growth speed of each leg could not always maintain

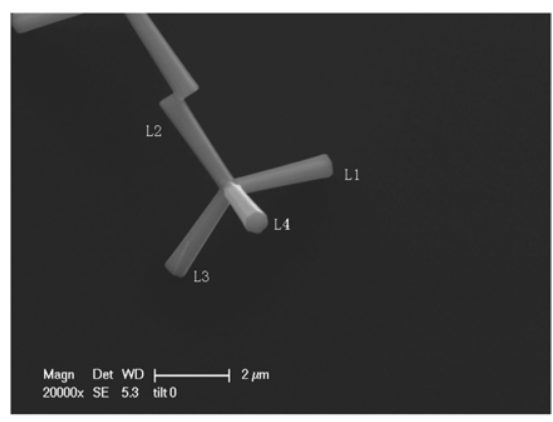

(a)

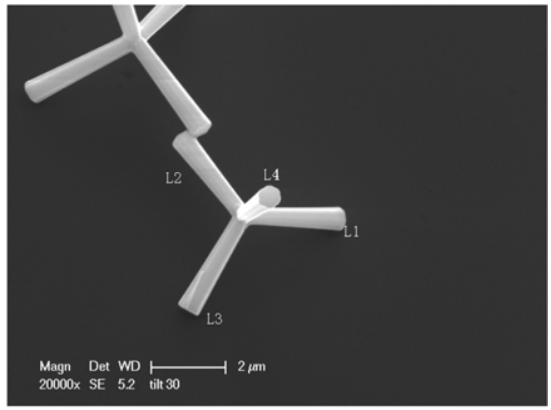

(b)

Figure 5. (a) A typical $\mathrm{ZnO}$ tetrapod and (b) SEM of the same tetrapod with the stage tilted $30^{\circ}$.

Table 1. The angles between every two legs of the $\mathrm{ZnO}$ tetrapod.

\begin{tabular}{|c|c|c|c|}
\hline L1 & L2 & L3 & L4 \\
\hline$\overline{\mathrm{L} 1}$ & $110.129^{\circ}$ & $113.591^{\circ}$ & 102.554 \\
\hline L2 & & $108.927^{\circ}$ & $113.879^{\circ}$ \\
\hline L3 & & & $107.735^{\circ}$ \\
\hline
\end{tabular}

the same accuracy for the fluctuation of the system. If one leg grows faster than its neighbouring legs in a certain period, the interface (actually the twin plane) between every two legs would be affected. This process would lead to the lattice mismatch of two neighbouring legs on the interface. Thus, the symmetry would be destroyed by the strain caused by the lattice mismatch.

\section{DFT simulation for the twin plane junction}

As shown in figure 3, the twin plane between every two legs is the vulnerable part of the structure. The crystal orientation of the twin plane is reported to be $(10 \overline{1} 3)[11,15]$.

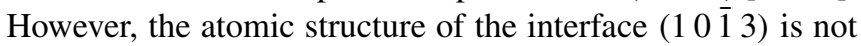
clear so far. Here the DFT calculation gives a stable model as shown in figure 6(a). Based on this model, we could estimate the energy needed to break the twin planes by the first principle calculation. The surface energy and interfacial energy calculations herein were performed within the DFT framework, using the program DMol3, a DFT programme within which the generalized gradient approximation (GGA) has been employed. The calculations were performed using the Perdew-Burke-Ernzerhof (PBE) exchange-correlation functional [16]. The schematic computational model of the surface is shown in figure $6(b)$. The surface energy 


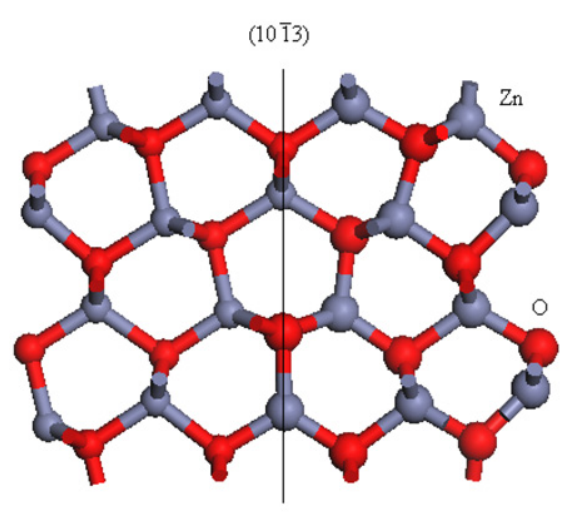

(a)

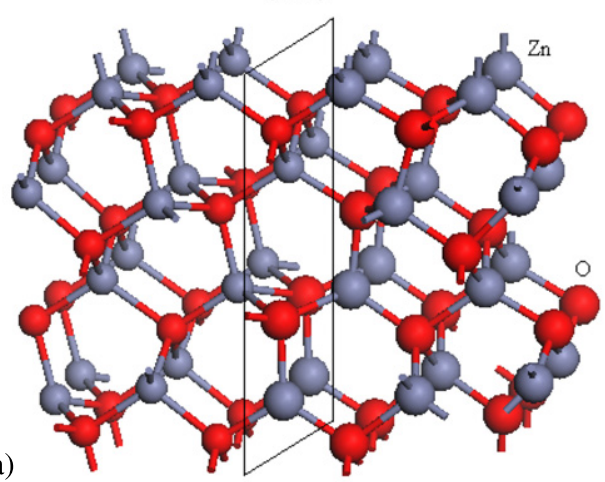

$(10 \overline{1} 3)$

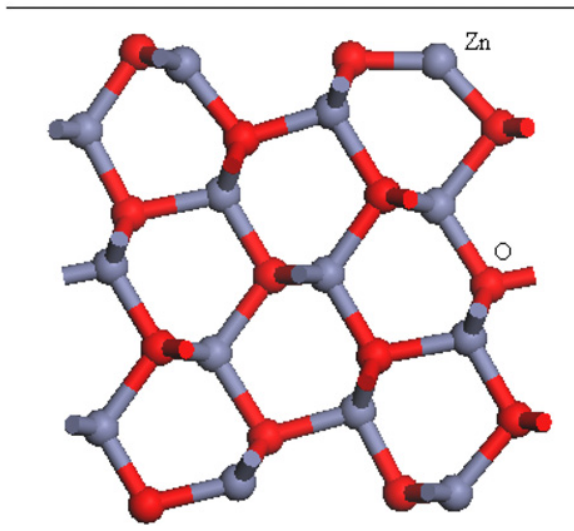

(b)

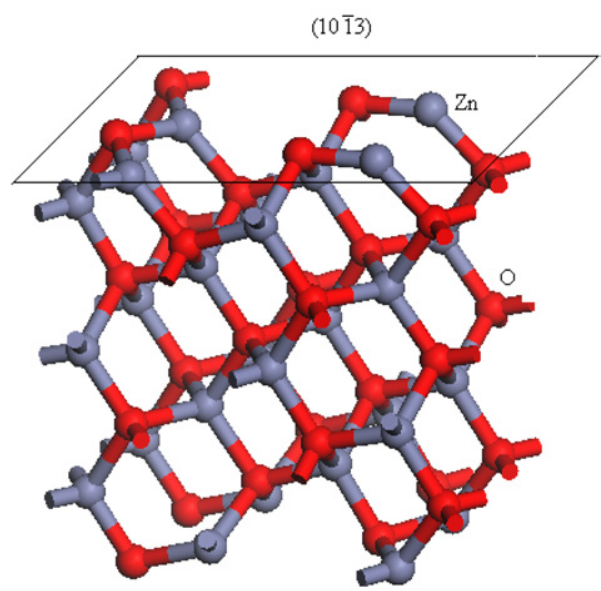

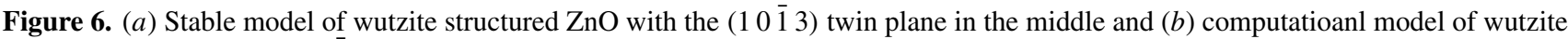

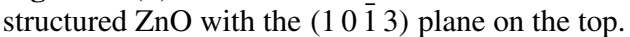

(This figure is in colour only in the electronic version)

was defined as the difference in energy between the perfect bulk crystal and the one with a certain surface, which was $\gamma_{(10 \overline{1})}=3.047 \mathrm{~J} \mathrm{~m}^{-2}$ according to the calculation. The calculated result for interfacial energy was $\gamma_{\text {interface }}=$ $0.443 \mathrm{~J} \mathrm{~m}^{-2}$, which was determined to be the difference between the energy of perfect bulk crystal and the one with interface [17].

Thus the energy needed to break the interface per area $E_{\mathrm{B}}$ was

$$
E_{\mathrm{B}}=2 \gamma_{(10 \overline{1} 3)}-\gamma_{\text {interface }}=5.651 \mathrm{~J} \mathrm{~m}^{-2} .
$$

Because the crystal faults usually accumulated on the interface between grains and the interface is imperfect, the actual interfacial energy would be significantly higher than the calculated result. So the actual energy needed to break the interface would be much lower than the result mentioned above.

\section{Conclusions}

In summary, the sample synthesized by the CVD method in this letter is of tetrapod shape and grows along the $\left(\begin{array}{llll}0 & 0 & 0 & 1\end{array}\right)$ direction. The twin planes are clearly observed at the junction of the tetrapods with SEM, which show that there is a twin plane between every two legs, and each leg has three lozenge twin planes. A twin plane model successfully explains the growth of tetrapod-like crystals and the structure of joint. Based on the DFT calculation, the stable atomic structure of interface is proposed. The energy needed to break the twin plane is determined to be $5.651 \mathrm{~J} \mathrm{~m}^{-2}$. These architectures are intriguing objects for further exploration of the physical properties and possible applications of advanced semiconductor nanodevices.

\section{Acknowledgments}

This work was jointly supported by the National Basic Research Program of China (973 Program, Grant No 2007CB310500) and the National Natural Science Foundation of China (NSFC, Grant Nos 10772180 and 10721202).

\section{References}

[1] Pan Z W, Dai Z R and Wang Z L 2001 Science 2911947

[2] Kong Y C, Yu D P, Zhang B, Fang W and Feng S Q 2001 Appl. Phys. Lett. 78407

[3] Schmidt O G and Eberl K 2001 Nature 410168

[4] Gudiksen M S, Lauhon L J, Wang J, Smith D C and Lieber C M 2002 Nature 415617

[5] Feng P, Wan Q and Wang T H 2005 Appl. Phys. Lett. 87213111 
[6] Gao P X, Ding Y, Mai W J, Hughes W L, Lao C S and Wang Z L 2005 Science 3091702

[7] He F Q and Zhao Y P 2006 Appl. Phys. Lett. 88193113

[8] He F Q and Zhao Y P 2006 J. Phys. D: Appl. Phys. 392105

[9] Shiojiri M and Kaito C 1981 J. Cryst. Growth 52173

[10] Nishio K, Isshiki T, Kitano M and Shiojiri M 1997 Phil. Mag. A 76889

[11] Zhang Z X, Yuan H J and Xie S S 2007 Appl. Phys. Lett. 90153116
[12] Ding Y, Gao P X and Wang Z L 2004 J. Am. Chem. Soc. 9126

[13] Ronning C and Shang N G 2005 J. Appl. Phys. 98034307

[14] Hu J Q, Bando Y and Golberg D 2005 Small 195

[15] Ding Y and Wang Z L 2007 Appl. Phys. Lett. 90153510

[16] Perdew J P, Burke K and Ernzerhof M 1996 Phys. Rev. Lett. 773865

[17] Van Bueren H G 1960 Imperfections in Crystals 2nd edn (Amsterdam: North-Holland) 